\title{
An application of metric cotype to quasisymmetric embeddings
}

\author{
Assaf Naor
}

\begin{abstract}
We apply the notion of metric cotype to show that $L_{p}$ admits a quasisymmetric embedding into $L_{q}$ if and only if $p \leq q$ or $q \leq p \leq 2$.
\end{abstract}

This note is a companion to [4]. After the final version of [4] was sent to the journal for publication I learned from Juha Heinonen and Leonid Kovalev of a long-standing open problem in the theory of quasisymmetric embeddings, and it turns out that this problem can be resolved using the methods of [4]. The argument is explained below. I thank Juha Heinonen and Leonid Kovalev for bringing this problem to my attention.

Let $\left(X, d_{X}\right)$ and $\left(Y, d_{Y}\right)$ be metric spaces. An embedding $f: X \rightarrow Y$ is said to be a quasisymmetric embedding with modulus $\eta:(0, \infty) \rightarrow(0, \infty)$ if $\eta$ is increasing, $\lim _{t \rightarrow 0} \eta(t)=0$, and for every distinct $x, y, z \in X$ we have

$$
\frac{d_{Y}(f(x), f(y))}{d_{Y}(f(x), f(z))} \leq \eta\left(\frac{d_{X}(x, y)}{d_{X}(x, z)}\right)
$$

We refer to [1] and the references therein for a discussion of this notion.

It was not known whether every two separable Banach spaces are quasisymetrically equivalent. This is asked in [6] (see problem 8.3.1 there). We will show here that the answer to this question is negative. Moreover, it turns out that under mild assumptions the cotype of a Banach space is preserved under quasisymmetric embeddings. Thus, in particular, our results imply that $L_{p}$ does not embed quasisymetrically into $L_{q}$ if $p>2$ and $q<p$. The question of determining when $L_{p}$ is quasisymetrically equivalent to $L_{q}$ was asked in [6] (see problem 8.3.3 there). We also deduce, for example, that the separable space $c_{0}$ does not embed quasisymetrically into any Banach space which has an equivalent uniformly convex norm.

We recall some definitions. A Banach space $X$ is said to have (Rademacher) type $p>0$ if there exists a constant $T<\infty$ such that for every $n$ and every $x_{1}, \ldots, x_{n} \in X$,

$$
\mathbb{E}_{\varepsilon}\left\|\sum_{j=1}^{n} \varepsilon_{j} x_{j}\right\|_{X}^{p} \leq T^{p} \sum_{j=1}^{n}\left\|x_{j}\right\|_{X}^{p} .
$$

where the expectation $\mathbb{E}_{\varepsilon}$ is with respect to a uniform choice of signs $\varepsilon=\left(\varepsilon_{1}, \ldots, \varepsilon_{n}\right) \in\{-1,1\}^{n}$. $X$ is said to have (Rademacher) cotype $q>0$ if there exists a constant $C<\infty$ such that for every $n$ and every $x_{1}, \ldots, x_{n} \in X$,

$$
\mathbb{E}_{\varepsilon}\left\|\sum_{j=1}^{n} \varepsilon_{j} x_{j}\right\|_{X}^{q} \geq \frac{1}{C^{q}} \sum_{j=1}^{n}\left\|x_{j}\right\|_{X}^{q} .
$$

We also write

$$
p_{X}=\sup \{p \geq 1: X \text { has type } p\} \quad \text { and } \quad q_{X}=\inf \{q \geq 2: X \text { has cotype } q\} .
$$


$X$ is said to have non-trivial type if $p_{X}>1$, and $X$ is said to have non-trivial cotype if $q_{X}<\infty$. For example, $L_{p}$ has type $\min \{p, 2\}$ and cotype $\max \{p, 2\}$ (see for example [5].

Theorem 1. Let $X$ be a Banach space with non-trivial type. Assume that $Y$ is a Banach space which embeds quasisymmetrically into $X$. Then $q_{Y} \leq q_{X}$.

Proof. Let $f: Y \rightarrow X$ be a quasisymmetric embedding with modulus $\eta$. Assume for the sake of contradiction that $X$ has cotype $q$ and that $p:=q_{Y}>q$. By the Maurey-Pisier theorem [2] for every $n \in \mathbb{N}$ there is a linear operator $T: \ell_{p}^{n} \rightarrow Y$ such that for all $x \in \ell_{p}^{n}$ we have $\|x\|_{p} \leq\|T(x)\|_{Y} \leq 2\|x\|_{p}$. For every integer $m \in \mathbb{N}$ consider the mapping $g: \mathbb{Z}_{m}^{n} \rightarrow X$ given by

$$
g\left(x_{1}, \ldots, x_{n}\right)=f \circ T\left(e^{\frac{2 \pi i x_{1}}{m}}, \ldots, e^{\frac{2 \pi i x_{n}}{m}}\right) .
$$

By Theorem 4.1 in [4] there exist constants $A, B>0$ which depend only on the type and cotype constants of $X$ such that for every integer $m \geq A n^{1 / q}$ which is divisible by 4 and every $h: \mathbb{Z}_{m}^{n} \rightarrow X$ we have

$$
\sum_{j=1}^{n} \mathbb{E}_{x}\left[\left\|h\left(x+\frac{m}{2} e_{j}\right)-h(x)\right\|_{X}^{q}\right] \leq B^{q} m^{q} \mathbb{E}_{\varepsilon, x}\left[\| h(x+\varepsilon)-\left.h(x)\right|_{X} ^{q}\right],
$$

where the expectations above are taken with respect to uniformly chosen $x \in \mathbb{Z}_{m}^{n}$ and $\varepsilon \in\{-1,0,1\}^{n}$ (here, and in what follows we denote by $\left\{e_{j}\right\}_{j=1}^{n}$ the standard basis of $\mathbb{R}^{n}$ ).

From now on we fix $m$ to be be the smallest integer which is divisible by 4 and $m \geq A n^{1 / q}$. Thus $m \leq 8 A n^{1 / q}$. For every $x \in \mathbb{Z}_{m}^{n}, j \in\{1, \ldots, n\}$ and $\varepsilon \in\{-1,0,1\}^{n}$ we have

$$
\frac{\|g(x+\varepsilon)-g(x)\|_{X}}{\left\|g\left(x+\frac{m}{2} e_{j}\right)-g(x)\right\|_{X}} \leq \eta\left(\frac{\left\|T\left(\sum_{k=1}^{n}\left(e^{\frac{\pi i \varepsilon_{k}}{m}}-1\right) e_{j}\right)\right\|_{Y}}{\left\|T\left(2 e_{j}\right)\right\|_{Y}}\right) \leq \eta\left(\frac{\pi n^{1 / p}}{m}\right) \leq \eta\left(\frac{\pi}{A} n^{\frac{1}{p}-\frac{1}{q}}\right) .
$$

Thus, using (1) for $g=h$ we see that

$$
\begin{aligned}
n \mathbb{E}_{\varepsilon, x}\|g(x+\varepsilon)-g(x)\|_{X}^{q} \leq \eta\left(\frac{\pi}{A} n^{\frac{1}{p}-\frac{1}{q}}\right)^{q} \sum_{j=1}^{n} \mathbb{E}_{x}\left\|g\left(x+\frac{m}{2} e_{j}\right)-g(x)\right\|_{X}^{q} & \\
& \leq \eta\left(\frac{\pi}{A} n^{\frac{1}{p}-\frac{1}{q}}\right)^{q}(8 A B)^{q} n \mathbb{E}_{\varepsilon, x}\|g(x+\varepsilon)-g(x)\|_{X}^{q} .
\end{aligned}
$$

Canceling the term $\mathbb{E}_{\varepsilon, x}\|g(x+\varepsilon)-g(x)\|_{X}^{q}$ we deduce that

$$
\eta\left(\frac{\pi}{A} n^{\frac{1}{p}-\frac{1}{q}}\right) \geq \frac{1}{8 A B}
$$

Since $p>q$ this contradicts the fact that $\lim _{t \rightarrow 0} \eta(t)=0$.

Using the same argument as in [4] (and noting that the snowflake embedding from [3] is a quasisymmetric embedding), we obtain the following complete answer to the question when $L_{p}$ embeds quasisymmetrically into $L_{q}$.

Corollary 2. For $p, q>0, L_{p}$ embeds quasisymmetrically into $L_{q}$ if and only if $p \leq q$ or $q \leq p \leq 2$. 


\section{References}

[1] J. Heinonen. Lectures on analysis on metric spaces. Universitext. Springer-Verlag, New York, 2001.

[2] B. Maurey and G. Pisier. Séries de variables aléatoires vectorielles indépendantes et propriétés géométriques des espaces de Banach. Studia Math., 58(1):45-90, 1976.

[3] M. Mendel and A. Naor. Euclidean quotients of finite metric spaces. Adv. Math., 189(2):451-494, 2004.

[4] M. Mendel and A. Naor. Metric cotype. 2005. To appear in Annals of Mathematics.

[5] V. D. Milman and G. Schechtman. Asymptotic theory of finite-dimensional normed spaces, volume 1200 of Lecture Notes in Mathematics. Springer-Verlag, Berlin, 1986. With an appendix by M. Gromov.

[6] J. Väisälä. The free quasiworld. Freely quasiconformal and related maps in Banach spaces. In Quasiconformal geometry and dynamics (Lublin, 1996), volume 48 of Banach Center Publ., pages 55-118. Polish Acad. Sci., Warsaw, 1999. 